\title{
Adaptive Interference Suppression in Multiuser Wireless OFDM Systems Using Antenna Arrays
}

\author{
Samir Kapoor, Member, IEEE, Daniel J. Marchok, and Yih-Fang Huang, Fellow, IEEE
}

\begin{abstract}
This paper considers the problem of mitigating fading and interference in wireless orthogonal frequency division multiplexing (OFDM) multiple access communication systems. Applications include cellular mobile radio, wireless local loop, and wireless local area networks. The effect of interchannel interference (ICI) arising from time-selective fading and frequency offsets and co-channel interference (CCI) is analyzed. A loop-timing method that enables a synchronous uplink between multiple mobile transceivers and a base-station is described. Adaptive antenna arrays are utilized at the base for uplink reception, and optimum array combining based on the maximum SINR criterion is used for each subchannel over slowly timevarying channels. For operation over fast time-varying channels, a novel two-stage adaptive array architecture that incorporates combined spatial diversity and constraint-based beamforming is presented. While ICI alone is most effectively overcome by spatial diversity, combined beamforming and diversity are most effective to combat $\mathrm{CCI}$ in the presence of fading. The overall method is suitable for real-time implementation and can be used in conjunction with traditional coding schemes to increase the link-margin.
\end{abstract}

Index Terms - Adaptive arrays, interference suppression, OFDM.

\section{INTRODUCTION}

$\mathbf{O}$ RTHOGONAL frequency division multiplexing (OFDM) has recently emerged as an attractive and powerful alternative to conventional modulation and multiple access schemes for wireless communications. Applications include wireless local loop, wireless local area networks, cellular, and personal communication services (PCS). Analogous to well-known time and code division multiple access systems, OFDM-based multiple access systems are also referred to as orthogonal frequency division multiple access (OFDMA) systems in the literature [18]. Recently, OFDM was chosen as the modulation scheme for the European digital audio broadcast (DAB) standard and the European terrestrial digital video broadcast (DVB-T) standard [2]. OFDM-based hybrid multiple access systems such as OFDM-time division multiple access (OFDM-TDMA) and multicarrier-code division multiple access (multicarrier-CDMA) are also being considered for next generation multiuser radio systems [2],

Manuscript received March 20, 1998; revised May 15, 1999. This work was supported in part by the National Science Foundation under Grant MIP9705173. The associate editor coordinating the review of this paper and approving it for publication was Dr. Sergios Theodoridis.

S. Kapoor is with NEC USA, Inc., Princeton, NJ 08540 USA.

D. J. Marchok is with Tellabs Research Center, Mishawaka, IN 46545 USA.

Y. F. Huang is with the Department of Electrical Engineering, University of Notre Dame, Notre Dame, IN 46556 USA.

Publisher Item Identifier S 1053-587X(99)09190-4.
[11], [15], [20]. A wireless OFDM multiple access system is considered in this paper in which one or more remote mobile transceivers communicate with a centralized base-station receiver.

The intelligent use of antenna arrays for mitigating fading and interference is often referred to as smart or intelligent antennas in the literature [6], [8], [9], [13]. Smart antenna systems are typically realized as switched beam or adaptive array antennas. Switched beam antennas use a fixed beamforming network to provide several output ports corresponding to beams in fixed directions. Signal levels in each beam are monitored and analyzed to switch the beams appropriately among different time or frequency channels, depending on the air-interface scheme. While actual operation is more complex, the basic idea is akin to classical switched diversity antenna systems. Adaptive arrays, on the other hand, electronically steer a phased array by weighting the amplitude and phase of signal at each element in response to changes in the propagation environment. For this purpose, there are a number of well-established methods available for weight adaptation and updating according to different optimization criteria [10], [26].

Wireless OFDM systems, like other air-interface schemes, are subject to the twin impairments of fading and co-channel interference (CCI). CCI occurs in cellular environments and when overlay networks are deployed. Fast or slowly timeselective fading also manifests itself as a source of interference in OFDM systems. In the absence of channel and implementation imperfections, each subchannel in an OFDM signal is exactly orthogonal to all other subchannels. ${ }^{1}$ However, timeselective fading diminishes interchannel orthogonality and leads to mutual interference between data-carrying OFDM subchannels, referred to as interchannel interference (ICI). Another major source of ICI is frequency offsets due to imperfect transceiver synchronization and phase noise. This problem exists to varying degrees in any practical system. The problem of ICI in OFDM systems has recently received some attention in the literature [5], [23], [25]. Increased frequency spacing of data carrying subchannels is an obvious solution, but it is clearly wasteful of bandwidth. The use of trellis-coded modulation and antenna diversity has been suggested in [21].

This paper focuses on the use of adaptive antennas at the base station to improve uplink OFDM reception. A synchronous uplink scheme based on loop-timing is proposed and described. This technique enables the base station to

\footnotetext{
${ }^{1}$ Subchannels are also referred to as frequency subcarriers, subsymbols, bins, or tones in the literature.
} 


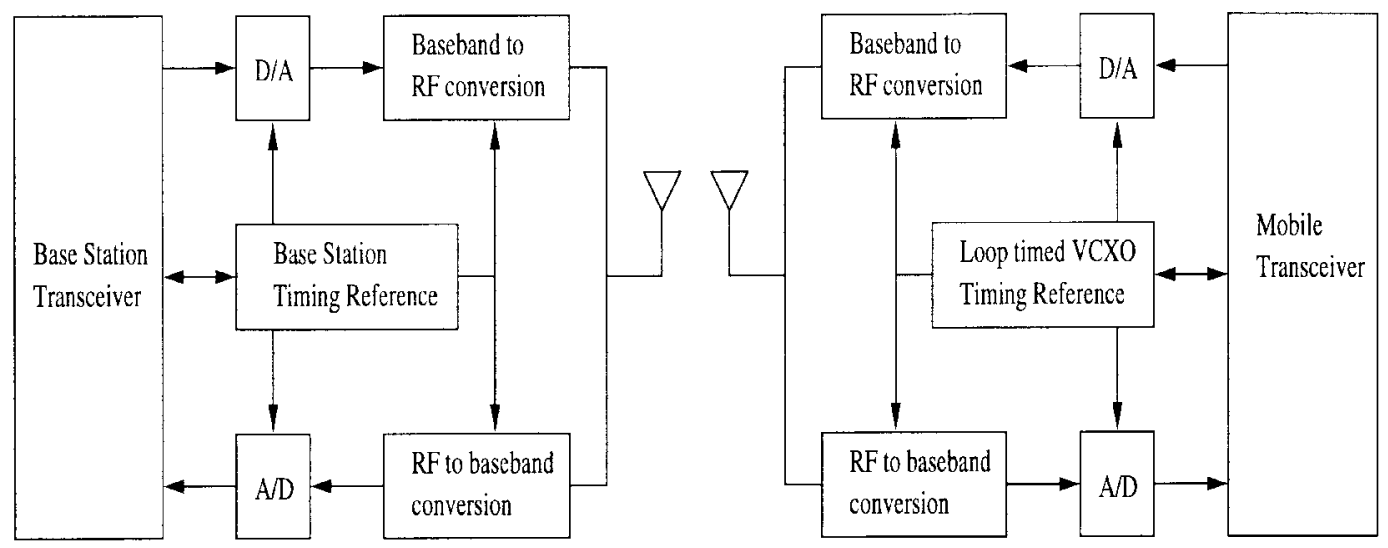

Fig. 1. Schematic diagram of loop-timed wireless OFDMA system.

synchronize the received OFDM signals from multiple users in the time and frequency domain. Adaptive array processing is carried out at baseband using demodulated frequency domain subsymbols. Transmission over both slow and fast time-varying fading channels is examined in the presence of ICI and CCI. It is well known that spatial diversity [using maximal ratio combining (MRC)] is most effective to combat ICI since it is spectrally distributed (also spatially distributed in a multiuser system) and may be lumped with the additive noise. However, this approach ceases to be optimal in the presence of co-channel interferers. For slowly time-varying channels, optimum array combining using the maximum signal-to-interference-plus-noise ratio (SINR) criterion is used. However, for fast time-varying channels, this approach becomes difficult, and an alternative two-stage adaptive array architecture is proposed. This allows for combined constraint-based beamforming and diversity combining for CCI suppression in the presence of additive white Gaussian noise (AWGN) and ICI. Overall, the use of adaptive array processing requires minimal bandwidth overhead and can be used in conjunction with traditional coding schemes to improve the link margin.

This paper is organized as follows. Section II describes the structure of an OFDMA system and formulates the mathematical model for transmission of OFDM signals over slow and fast time-varying channels and in the presence of frequency offsets. Section III introduces the use of antenna array for uplink reception and describes the proposed array combining algorithms. Numerical results appear in Section IV, and the paper is concluded in Section V.

\section{PROBlem Formulation}

\section{A. Uplink Multiple Access Using OFDM}

In an OFDMA system, the entire uplink bandwidth processed by a base station is (dynamically) allocated among a group of users. Unlike uplink TDMA systems in which remote units transmit in bursts in specified periodic timeslots, uplink OFDMA can be made synchronous using the method of loop timing [12]. Fig. 1 depicts the base station and mobile transceivers in a loop-timed OFDMA system with emphasis on the synchronization subsystem. In this method, each mobile transceiver first synchronizes itself to the base station on the downlink and then derives its uplink transmitter timing reference from the recovered downlink clock. To facilitate the former task, the base station embeds pilot tones in the transmitted downlink signal, which are utilized by the remote receiver to "lock-on" to the base's timing reference. To overcome frequency selective fading across the signal bandwidth, multiple pilots can be used. While conventional baseband digital phase-locked-loops can be used for operation over slowly time-varying channels, other techniques are used for frequency acquisition and tracking for operation over fast time-varying channels; see, e.g., [3] and [12]. The local timing reference for mobile transceivers are usually derived from a voltage controlled crystal oscillator (VCXO), which provides the timing reference for the receiver $\mathrm{A} / \mathrm{D}$, transmitter D/A, and all radio frequency (RF) circuitry. Frequency offsets between the receive and transmit symbol clock occur due to nonidealities in the remote transceiver VCXO's, possibly of the order of several parts per million (ppm).

Two main synchronization tasks are performed by the base station transceiver-frequency synchronization and time synchronization. Frequency synchronization ensures that the base station receiver can use a single FFT to simultaneously demodulate all active user bins without mutual interference. If this were not the case, the FFT response for bins belonging to any one user-set would not exhibit frequency-domain nulls at the FFT output of bins for other users and, consequently, appear as ICI. Frequency synchronization also compensates for oscillator and Doppler drifts since they manifest as ICI. While it is impossible to compensate and track for these changes perfectly, their effect can be reduced to a tolerable level below the ambient noise floor.

Time synchronization (or OFDM symbol alignment) is necessary to ensure that the symbol boundaries of different users are aligned so that they maintain orthogonality. This measurement and adjustment of the time delays of various user signals is also called ranging and must be carried out when multipath is present. Owing to the presence of the cyclic prefix (Section II-B), the resolution required for time synchronization can be relaxed (by a few samples) by selecting 


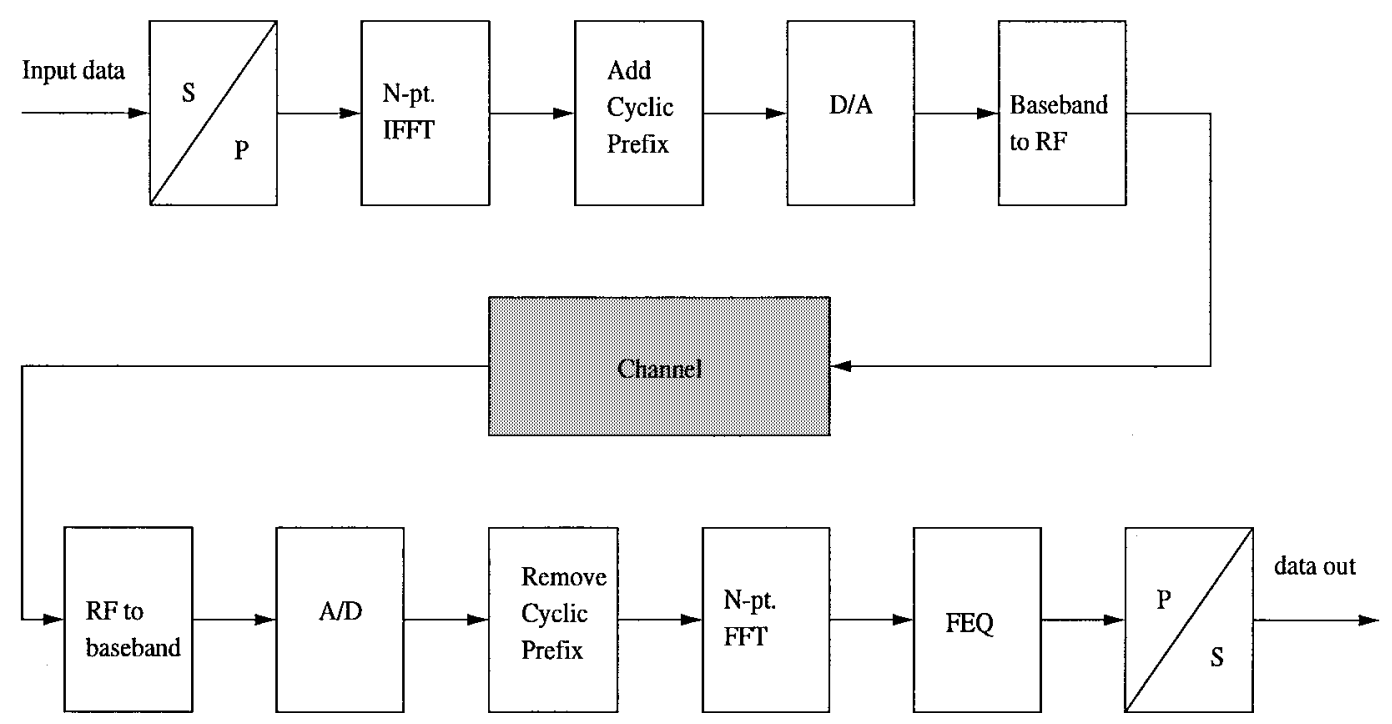

Fig. 2. Schematic diagram of conventional OFDM transmitter and receiver.

the cyclic prefix length to be greater than the maximum cell delay spread. This incurs a proportionate, but minor, loss in bandwidth efficiency. Techniques for time synchronization include use of null (or other pilot) symbols and/or pilot bins; see, e.g., [7]. In a dynamic environment, symbol alignment has to be frequently updated, e.g., on a frame-by-frame basis. Delay spreads of several microseconds can be tolerated (see parameters in Section IV) leading to feasibility of operation within a radius of few miles from the base (macro-cells) [7].

In the rest of this paper, it is assumed that the initial tasks of carrier frequency and time synchronization have been completed (the effect of residual frequency offsets is examined in Section III). This enables the base station receiver to demodulate received baseband signals from all users with a single FFT. The base station is also responsible for all bandwidth management functions to provide each unit with shared access to the uplink channel, depending on the number of active users, interference environment, required bit rates, etc.

\section{B. OFDM Transmission Model}

Consider a discrete time OFDM system model in which $N$ orthogonal sinusoids are transmitted at each symbol time. The $N$ subchannels are partitioned among a total of $L$ independent users in nonoverlapping sets of $K \triangleq N / L$ (assumed integer) subchannels for each user. Without loss of generality, a complex baseband representation is used for all signals. Thus, the normalized transmitted signal from the $l$ th user is given by

$$
\begin{aligned}
s_{l}(n)=\frac{1}{\sqrt{N}} \sum_{k \in \mathcal{S}_{l}} a(k) e^{j(2 \pi / N) k n} \\
\quad n \in[0, N-1], \quad l \in[0, L-1]
\end{aligned}
$$

where $j=\sqrt{-1} ; a(k)$ is the $k$ th frequency domain subsymbol typically selected from a quadrature amplitude modulation (QAM) constellation, and $\mathcal{S}_{l}$ denotes the set of subchannel indices belonging to the $l$ th user. Each symbol is prefixed with a cyclic prefix (CP) or guard time. This eliminates intersymbol

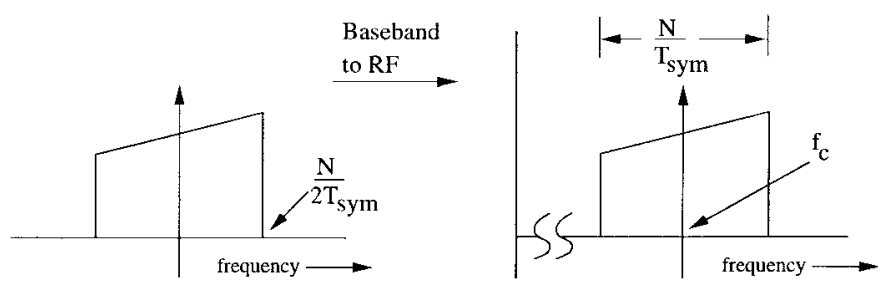

Fig. 3. Spectrum of OFDM signals at baseband and RF carrier frequency $f_{c}$.

interference and preserves orthogonality between bins when the $\mathrm{CP}$ duration $\left(T_{C P}\right)$ is longer than the channel impulse response (CIR) duration $\left(T_{C I R}\right)$. In addition, by appropriately selecting the transmitted samples of the $\mathrm{CP}$, the transmitted signal appears periodic to the channel resulting in simplified frequency-domain equalization [4], [5]. This is is done by setting

$$
s_{l}(-j)=s_{l}(N-j), \quad j \in[1, G], l \in[0, L-1]
$$

where $G$ is chosen such that $T_{C P} \geq T_{C I R}$, i.e., $G \geq U$. In addition, the $\mathrm{CP}$ can also be utilized for synchronization purposes [3]. The receiver discards a block of $G$ samples (appropriately indexed according to the time synchronization procedure) before demodulation, and it is sufficient to consider each symbol independently (Fig. 2).

The discrete-time sequence $\left\{s_{l}(n)\right\}$ is applied at a suitably chosen rate of $f_{s}$ samples/s to a D/A converter that converts it into an analog signal as $\Sigma_{n} s_{l}(n) h_{d a}\left(t-n T_{s}\right)$, where $h_{d a}(t)$ denotes the D/A pulse shape, and $T_{s}=1 / f_{s}$. The D/A output is typically further filtered by an antialias filter with impulse response denoted by $h_{a a}(t)$. Thus, the analog signal at the output of the transmitter is given by $\Sigma_{n} s_{l}(n)\left(h_{d a} \star\right.$ $\left.h_{a a}\right)\left(t-n T_{s}\right)$, where $\star$ denotes linear convolution. This signal encounters a time-selective multipath fading channel impulse response and impinges on the receiver front end, where it undergoes front-end (antialias) filtering and A/D conversion. In the following, for the $l$ th user, the composite sampled impulse response due to the transmit D/A, transmit antialias filter, multipath CIR, receiver antialias filter, and receiver A/D is 
denoted by $h_{u, l}(n)$ [response at time $n$ due to the impulse $\delta(n-u)]$. The multipath CIR is the time-varying portion. In addition, assume that the maximum duration of $h_{u, l}(\cdot)$ is $T_{C I R}=U / f_{s}$. Thus, the received signal from the $l$ th user after passing through the channel is given by

$$
x_{l}(n)=\sum_{u=n}^{n-U+1} h_{u, l}(n) s_{l}(u), \quad l \in[0, L-1] .
$$

Note that slowly time-varying channels may be considered to be time invariant over a symbol period leading to $h_{u, l}(n)=$ $h_{l}(n-u)$. Using (2.1) in (2.2)

$$
\begin{gathered}
x_{l}(n)=\frac{1}{\sqrt{N}} \sum_{u=0}^{U-1} h_{n-u, l}(n) \sum_{k \in \mathcal{S}_{l}} a(k) e^{j(2 \pi / N) k(n-u)} \\
n \in[0, N-1], l \in[0, L-1] .
\end{gathered}
$$

The overlapping uplink signal at the base station from all users is given by

$$
r(n)=\sum_{l=0}^{L-1} x_{l}(n)+v(n)+z(n)
$$

where $v(n)$ denotes discrete-time AWGN samples with variance $\sigma_{v}^{2}$, and $z(n)$ denotes discrete-time CCI samples (both after receiver front-end filtering and A/D conversion). After discarding the $\mathrm{CP},(2.3)$ can be written compactly in matrix form for an entire symbol as

$$
\mathbf{r}=\sum_{l=0}^{L-1} \sum_{u=0}^{U-1} \mathbf{H}_{u, l} \mathbf{T}_{l} \mathbf{D}_{u, l} \mathbf{a}_{l}+\mathbf{v}+\mathbf{z}
$$

where $\quad \mathbf{r}=[r(0), r(1), \cdots, r(N-1)]^{T}$ is the received signal vector; $\mathbf{a}_{l} \in \mathcal{C}^{K}$ denotes the column vector of frequency domain subsymbols from the lth user; $\mathbf{v}=[v(0), v(1), \cdots, v(N-1)]^{T} \in \mathcal{C}^{N}$; $\mathbf{z}=[z(0), z(1), \cdots, z(N-1)]^{T} \in \mathcal{C}^{N} ; \mathbf{H}_{u, l}=$ $\operatorname{diag}\left(\left[h_{-u, l}(0), h_{-u+1, l}(1), \cdots, h_{-u+N-1, l}(N-1)\right]\right) \in \mathcal{C}^{N \times N}$ denotes the diagonal CIR matrix for the $l$ th user and $u$ th delay; $\mathbf{D}_{u, l} \in \mathcal{C}^{K \times K}$ denotes a diagonal matrix of phase delays whose element corresponding to the $k$ th subchannel is given by $e^{-j(2 \pi / N) k u}$, and $\mathbf{T}_{l} \in \mathcal{C}^{N \times K}$ denotes the inverse-DFT modulating matrix whose column corresponding to the $k$ th subchannel is given by $\mathbf{t}_{l, k}=(1 / \sqrt{N})\left[1, e^{j(2 \pi \cdot N) k}, \cdots, e^{j(2 \pi / N) k(N-1)}\right]^{T}$.

\section{Slowly Time-Varying Channel}

We begin by considering a slowly varying fading channel in which the impulse response may be taken to be time-invariant over a symbol period. This case illustrates the demodulating procedure and will also serve as a starting point for the rest of the analysis. Thus, $\mathbf{H}_{u, l}=h_{l}(u) \mathbf{I}_{N}$, where $h_{l}(u)$ is the $u$ th impulse response coefficient. Using (2.4), the $p$ th subchannel belonging to, say, the $l$ th user is demodulated as

$$
y(p)=\mathbf{t}_{l, p}^{H} \mathbf{r}=\alpha_{s}(p) a(p)+\hat{v}(p)+\hat{z}(p), \quad p \in \mathcal{S}_{l}
$$

where

$$
\alpha_{s}(p)=\sum_{u=0}^{U-1} h_{l}(u) e^{-j(2 \pi / N) u p}
$$

and $\hat{v}(p)$ and $\hat{z}(p)$ denote the post-demodulation residual noise and CCI, respectively. Thus, there is no fading induced ICI in this case. There are several methods for frequencydomain equalization ranging from no equalization at all (for instance, using differential-phase-shift-keying schemes such as D-QPSK) [4] to sophisticated time-frequency adaptive filtering algorithms [19], [23]. The former are usually sufficient for slowly time-varying channels, whereas the latter are used in more demanding environments.

\section{Fast Time-Varying Channel}

As above, using (2.4), the pth subchannel belonging to, say, the $l$ th user is demodulated as

$$
\begin{aligned}
y(p) & =\sum_{l=0}^{L-1} \sum_{u=0}^{U-1} \mathbf{t}_{l, p}^{H} \mathbf{H}_{u, l} \mathbf{T}_{l} \mathbf{D}_{u, l} \mathbf{a}_{l}+\mathbf{t}_{l, p}^{H}(\mathbf{v}+\mathbf{z}) \\
& =\alpha_{f}(p) a(p)+i_{f}(p)+\hat{v}(p)+\hat{z}(p) \quad p \in \mathcal{S}_{l}
\end{aligned}
$$

where

$$
\begin{aligned}
\alpha_{f}(p)= & \frac{1}{N} \sum_{u=0}^{U-1} \sum_{n=0}^{N-1} h_{n-u, l}(n) e^{-j(2 \pi / N) u p} \\
i_{f}(p)= & \frac{1}{N} \sum_{l=0}^{L-1} \sum_{\substack{k \in \mathcal{S}_{l} \\
k \neq p}} \sum_{u=0}^{U-1} \sum_{n=0}^{N-1} h_{n-u, l}(n) \\
& \cdot e^{j(2 \pi / N) n(k-p)} e^{-j(2 \pi / N) u k} a(k) .
\end{aligned}
$$

It is straightforward to show that the variance of $\hat{v}(p)$ equals $\sigma_{v}^{2}$, and the post-demodulation CCI variance is given by

$$
\begin{aligned}
\sigma_{z}^{2}(p) & =E\left[|\hat{z}(p)|^{2}\right] \\
& =\sum_{n=-(N-1)}^{N-1}(1-|n| / N) r_{z}(n) e^{-j(2 \pi / N) n p} \\
& =\left.P_{z}(w) \star \frac{\sin ^{2}(N w / 2)}{N \sin ^{2}(w / 2)}\right|_{w=(2 \pi p / N)}
\end{aligned}
$$

where

$E[\cdot] \quad$ ensemble expectation;

$r_{z}(\cdot) \quad$ CCI discrete time auto-correlation function;

$P_{z}(w)$ CCI power spectral density (PSD).

Thus, for a given, say, $p$ th bin, the post-demodulation CCI is given by the convolution of the CCI PSD with a $\operatorname{sinc}^{2}(\cdot)$ function evaluated at the corresponding angular frequency. Each demodulated subsymbol is now corrupted by ICI from all other subsymbols. The effect of ICI is damaging for even small values of Doppler spreads and frequency offsets and can severely limit the bit error rate performance [4], [5], [21]. Such an effect is depicted in Fig. 6, where the residual interference from an equal relative power $(0 \mathrm{dBc})$ tone interferer is plotted. If the tone interferer is $f_{t} \mathrm{~Hz}$ away from the center of a particular subchannel, the normalized frequency offset is $f_{t} / f_{\text {bin }}$. This shape of the spectral leakage function is a direct consequence of using the discrete Fourier transform as the OFDM modulation basis function. 


\section{E. Effects of Frequency Offsets}

Consider now the effect of time-varying random frequency offsets due to imperfect transceiver synchronization and phase noise. This issue becomes particularly important when the synchronization problem is aggravated by fast time-varying channels or for systems that are sensitive to power and complexity considerations. Let the normalized frequency offset for the $l$ th user be denoted by $\eta_{l}=f_{o f f, l} / f_{\text {bin }}$, where $f_{o f f, l}$ denotes the frequency offset in Hertz and the interchannel spacing $f_{b i n}=f_{s} / N$. Each symbol time, $\eta_{l}$ is modeled as a realization of an independent uniformly distributed random variable in the interval $\left[-\eta_{\max }, \eta_{\max }\right]$. Thus, the demodulating vector with frequency offset is given by $\mathbf{J}\left(\eta_{l}\right) \mathbf{t}_{l, p}$, where $\mathbf{J}\left(\eta_{l}\right) \in \mathcal{C}^{N \times N}$ denotes a diagonal offset matrix with $n$th element given by $e^{j\left((2 \pi / N) n \eta_{l}+\beta\right)}$, where $\beta \in[-\pi, \pi]$ denotes a phase offset varying from symbol to symbol. Thus

$$
\begin{aligned}
y(p)= & \sum_{l=0}^{L-1} \sum_{u=0}^{U-1} \mathbf{t}_{l, p}^{H} \mathbf{J}^{H}\left(\eta_{l}\right) \mathbf{H}_{u, l} \mathbf{T}_{l} \mathbf{D}_{u, l} \mathbf{a}_{l} \\
& +\mathbf{t}_{l, p}^{H} \mathbf{J}^{H}\left(\eta_{l}\right)(\mathbf{v}+\mathbf{z}) \\
= & \alpha_{f, \eta}(p) a(p)+i_{f, \eta}(p)+\hat{v}_{\eta}(p)+\hat{z}_{\eta}(p) \\
& p \in \mathcal{S}_{l}
\end{aligned}
$$

where

$$
\begin{aligned}
& \alpha_{f, \eta}(p)=\frac{1}{N} \sum_{u=0}^{U-1} \sum_{n=0}^{N-1} h_{n-u, l}(n) \\
& \cdot e^{j\left((2 \pi / N) n \eta_{l}+\beta\right)} e^{-j(2 \pi / N) u p} \\
& i_{f, \eta}(p)=\frac{1}{N} \sum_{l=0}^{L-1} \sum_{\substack{k \in \mathcal{S}_{l} \\
k \neq p}} \sum_{u=0}^{U-1} \sum_{n=0}^{N-1} h_{n-u, l}(n) \\
& \cdot e^{j\left((2 \pi / N) n\left(k-p+\eta_{l}\right)+\beta\right)} e^{-j(2 \pi / N) u k} a(k) .
\end{aligned}
$$

The SINR for the $p$ th bin is defined as

$$
\operatorname{SINR}(p)=\frac{E\left[\left|\alpha_{f, \eta}(p) a(p)\right|^{2}\right]}{E\left[\left|i_{f, \eta}(p)+\hat{z}_{\eta}(p)\right|^{2}\right]+\sigma_{v}^{2}}
$$

Assuming a wide sense stationary uncorrelated scattering (WSSUS) multipath fading model and a Rayleigh fading Doppler spectrum [14], expressions for signal and interference powers in (2.8) appear in the Appendix.

\section{ReCEPTION With ANTENNA ARRAy ReCEIVER}

In conventional single-antenna wireless OFDM receivers, FFT-based demodulation is generally followed by a frequency domain equalizer (FEQ) and subsequent mapping of the equalized frequency domain subsymbols to bits (Fig. 2). In an adaptive array OFDM receiver, demodulator outputs from each subarray element are fed into a bank of array combiners where a separate array combining vector is used for each subchannel. We propose the use of the maximum SINR criterion and constraint-based beamforming [6], [26] for weight adaptation

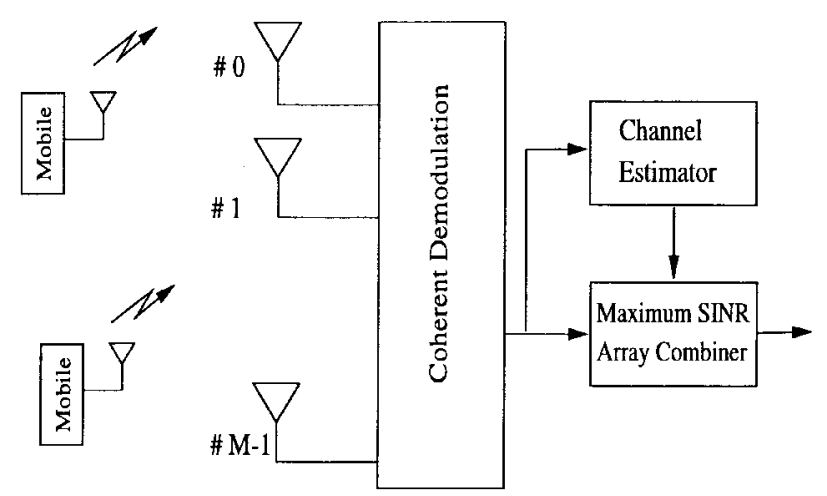

Fig. 4. Schematic of OFDM array receiver for slow time-varying fading channels with maximum SINR array combining.

under appropriate channel conditions, as described later in this section (Fig. 4). In the former method, channel estimation is necessary, and antennas are spaced far enough apart to obtain independent fading between elements. To maximize the SINR at the output of the array, the optimum weight vector balances diversity and interference suppression. In the latter approach, antenna elements are spaced sufficiently close to prevent spatial aliasing (i.e., grating lobes) and facilitate the application of constraints, such as those derived from estimates of direction-of-arrival (DOA) of impinging signals.

\section{A. Reception over Slowly Time-Varying Channels}

For a given data rate and bandwidth, the ratio of symbol rate to fading rate (or Doppler spread) in OFDM is much smaller compared with single-carrier systems. For example, with a typical OFDM symbol rate of $4 \mathrm{KHz}$ and with a Doppler spread of $200 \mathrm{~Hz}$, the ratio is 20 (the same ratio for the IS-136 TDMA system having a $24.3-\mathrm{KHz}$ symbol rate is about six times greater). For the maximum SINR method to be effective, the temporal averaging used to estimate the noiseplus-interference statistics must be done over a time duration much smaller than the duration over which the fading changes significantly. Channel estimates for each antenna element are also required. Moreover, this procedure has to be carried out separately for every data carrying bin. Thus, this approach is suitable only for OFDM systems with slow time-varying fading, for example, in low mobility scenarios.

Thus, we utilize statistically optimum array combining based on the maximum SINR criterion for operation over slowly fading channels. With a $M$ element array receiver depicted in Fig. 4, let the CIR matrix for the $m$ th element user be denoted by $\mathbf{H}_{u, l}^{(m)}=h_{l}^{(m)}(u) \mathbf{I}_{N}$. The array elements are spaced sufficiently apart to obtain uncorrelated fading. The corresponding received signal is given by

$$
\mathbf{r}^{(m)}=\sum_{l=0}^{L-1} \sum_{u=0}^{U-1} h_{l}^{(m)}(u) \mathbf{T}_{l} \mathbf{D}_{u, l} \mathbf{a}_{l}+\mathbf{v}^{(m)}+\sum_{e} \mathbf{z}_{e}^{(m)}
$$

where $\mathbf{z}_{e}^{(m)}$ denotes the eth CCI component received at the $m$ th subarray. Thus, the demodulated signal at the output of 
the $m$ th element is given by

$$
\begin{aligned}
y^{(m)}(p)= & \sum_{l=0}^{L-1} \sum_{u=0}^{U-1} \mathbf{t}_{l, p}^{H} \mathbf{J}^{H}\left(\eta_{l}\right) h_{l}^{(m)}(u) \mathbf{T}_{l} \mathbf{D}_{u, l} \mathbf{a}_{l} \\
& +\mathbf{t}_{l, p}^{H} \mathbf{J}^{H}\left(\eta_{l}\right)\left(\mathbf{v}^{(m)}+\sum_{e} \mathbf{z}_{e}^{(m)}\right) \\
= & \alpha_{s, \eta}^{(m)}(p) a(p)+i_{s, \eta}^{(m)}(p)+\hat{v}_{\eta}^{(m)}(p)+\sum_{e} \hat{z}_{e, \eta}^{(m)}(p)
\end{aligned}
$$

where $\alpha_{s, \eta}(p)$ and $i_{s, \eta}^{(m)}(p)$ are given by setting $h_{n-u, l}(n)$ to $h_{l}^{(m)}(u)$ in (2.7). Denoting the vector of demodulated $p$ th subchannel outputs from all $M$ elements as $\mathbf{y}(p)=$ $\left[y^{(0)}(p), y^{(1)}(p), \cdots, y^{(M-1)}(p)\right]^{T}$, the estimated $p$ th subsymbol is obtained as $\hat{a}(p)=\mathbf{w}^{H}(p) \mathbf{y}(p)$. The optimum weight vector that maximizes the SINR at the array output (referred to hereafter as OSINR) is $\mathbf{w}_{\text {opt }}(p)=\gamma(p) \mathbf{R}_{y}^{-1}(p) \Lambda(p)$, where $\gamma(p)$ is a constant (not affecting the OSINR); $\mathbf{R}_{y}(p) \in$ $\mathcal{C}^{M \times M}$ is the received data covariance matrix, and $\Lambda(p)=$ $\left[\alpha_{s, \eta}^{(0)}(p), \alpha_{s, \eta}^{(1)}(p), \cdots \alpha_{s, \eta}^{(M-1)}(p)\right]^{T}$ is the propagation vector for the $p$ th bin [26]. Simulation results with various parameters are presented in Section IV. $\mathbf{R}_{y}(p)$ and $\Lambda(p)$ are estimated by periodic pilot subsymbols inserted in the each active bin. A number of techniques can be used for channel estimation $[\Lambda(p)]$ using data-directed (i.e., training sequence) or decisiondirected operation, taking into account the time-frequency dispersive characteristics of the channel; see, e.g., [18], [19], [23], and the references therein.

\section{B. Reception Over Fast Time-Varying Channels}

In this case, constraint-based beamforming is used with the constraints chosen such that their rate of change is significantly slower than the data rate. This approach also allows for flexible and general constraints, albeit at the expense of higher computation required for their generation. In the sequel, DOA-based constraints are used to exploit angle diversity. To enable simultaneous exploitation of spatial and angle diversity, the base station array is partitioned into multiple subarrays (Fig. 5). While the elements within each subarray are closely spaced, the individual subarrays are spaced far apart. This allows for combined use of angle diversity (via constraintbased beamforming in each subarray) and spatial diversity (via diversity combining of all subarray outputs). Thus, as depicted in Fig. 5, consider a base station antenna array configuration comprised of $M$ subarrays, each with $S$ elements, totaling $M S$ elements. The relationship between angles of arrival, beamwidth of arriving signals, and antenna spacing has been explored in [22]. For an interelement spacing of $\rho$ and narrowband signal wavelength $\lambda$, the fading experienced at adjacent sensors is almost perfectly correlated for sufficiently small values of $\rho / \lambda$ (such as 0.5 or less) and angle spreading (around $5^{\circ}-10^{\circ}$ or less). Thus, the interelement spacing within each subarray $\rho$ is chosen to facilitate beamforming. A large inter subarray spacing, on the other hand, is chosen to obtain a spatial diversity gain. For example, a spacing of $5 \lambda$ to $10 \lambda$ or more is regarded to be adequate for obtaining sufficiently low fading correlation [14], [22]. Note that conventional

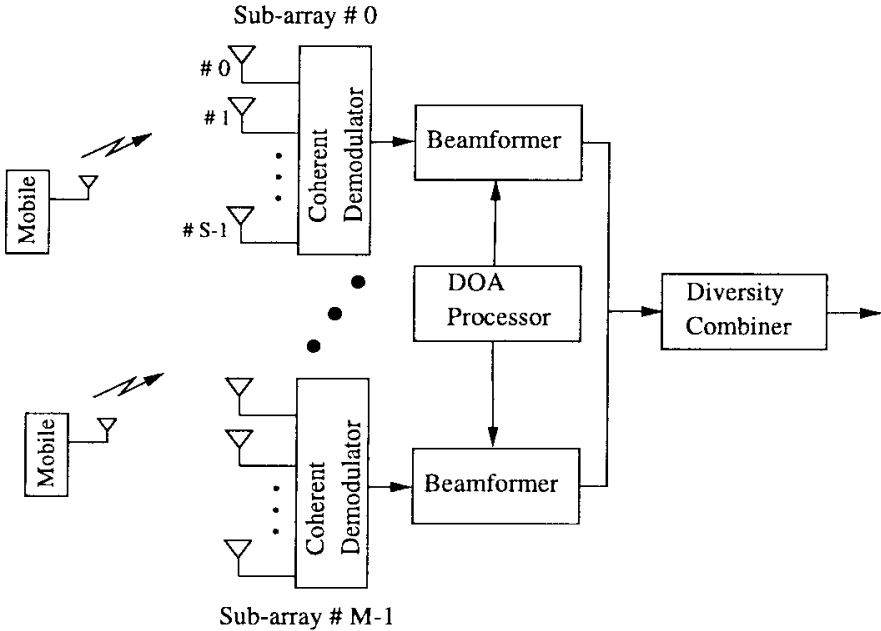

Fig. 5. Schematic of OFDM array receiver for fast time-varying fading channels with two stage array combining.

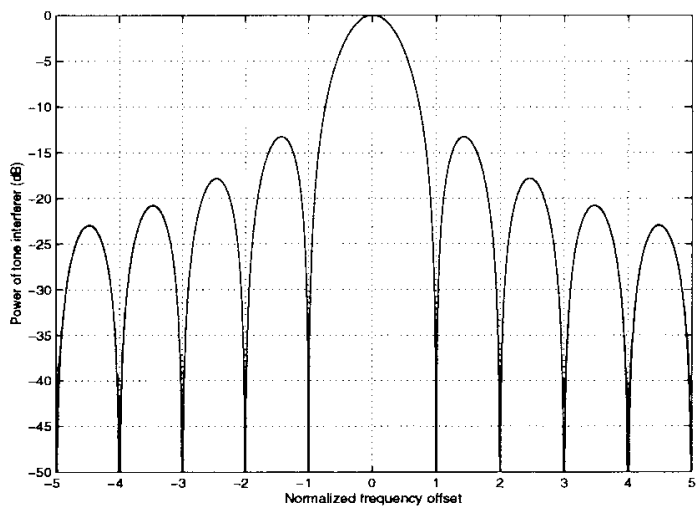

Fig. 6. Spectral leakage from $0 \mathrm{dBc}$ co-channel tone interferer.

beamforming only or diversity combining only arrays are special cases of this configuration with $M=1$ and $S=1$, respectively. If MRC is used for combining outputs from different subarrays in the second stage, a separate FEQ is not needed. On the other hand, if switched diversity combining is used, an FEQ is still required. Thus, if channel estimation is to be eliminated, switched diversity is appropriate in conjunction with differential signaling.

For typical cellular systems, the rate of change of DOA's is much lower than the symbol rate allowing for the use of only a few pilot subchannels to obtain DOA estimates. Moreover, each of these constraints can be utilized for multiple bins, thus greatly reducing the total computational burden for constraint generation. Another key advantage of DOA-based beamforming is that since DOA information is independent of carrier frequency, the information can be reused for downlink beamforming as well. See [1], [6], [9], [13], and [24] for DOA estimation algorithms employed to estimate and track directions of desired and interfering signals specifically in mobile radio environments. In addition to conventional algorithms, DOA estimation techniques based on time-differenceof-arrival and multiple base station triangulation are also emerging [17], and the resulting information can also be utilized for other tasks such as mobile handoffs and geolocation. 
Extending the notation of the previous section, let the CIR matrix for the $m$ th subarray be denoted by $\mathbf{H}_{u, l}^{(m)}$. The received signal at the sth element of the $m$ th subarray in the presence of spatially directional desired signals and CCI is given by

$$
\begin{aligned}
\mathbf{r}^{(m, s)}= & \sum_{l=0}^{L-1} c_{l}^{(m, s)} \sum_{u=0}^{U-1} \mathbf{H}_{u, l}^{(m)} \mathbf{T}_{l} \mathbf{D}_{u, l} \mathbf{a}_{l}+\mathbf{v}^{(m, s)} \\
& +\sum_{e} c_{e}^{(m, s)} \mathbf{z}_{e}^{(m, s)}
\end{aligned}
$$

where $c_{l}^{(m, s)}$ and $c_{e}^{(m, s)}$ denote the multiplicative factors that can be factored out in the sth sensor's response of the $m$ th subarray with respect to the reference sensor $(s=0)$ for the $l$ th user and eth CCI component, respectively. For instance, if the signals are assumed to emerge from point sources, $c_{l}^{(m, s)}=$ $e^{j s \phi_{p}} ; p \in \mathcal{S}_{l}$ and $c_{e}^{(m, s)}=e^{j s \phi_{e}}$, where $\phi_{p}$ and $\phi_{e}$ denote the spatial DOA's given by $\phi_{p}=2 \pi(\rho / \lambda) \sin \left(\theta_{p}\right), p \in \mathcal{S}_{l}$ and $\phi_{e}=2 \pi(\rho / \lambda) \sin \left(\theta_{e}\right)$ corresponding to DOA's $\theta_{p}$ and $\theta_{e}$ of the particular desired signal and CCI, respectively. If the sources are assumed to be in the array far field, the directions can be assumed to be unchanged with respect to each subarray's reference element. Thus, the demodulated signal at the output of the $(m, s)$ th element is given by

$$
\begin{aligned}
y^{(m, s)}(p)= & \sum_{l=0}^{L-1} \sum_{u=0}^{U-1}\left\{\mathbf{t}_{l, p}^{H} c_{l}^{(m, s)} \mathbf{J}^{H}\left(\eta_{l}\right) \mathbf{H}_{u, l}^{(m)} \mathbf{T}_{l} \mathbf{D}_{u, l} \mathbf{a}_{l}\right. \\
& +\mathbf{t}_{l, p}^{H} \mathbf{J}^{H}\left(\eta_{l}\right)\left(\mathbf{v}^{(m, s)}+\sum_{e} c_{e}^{(m, s)} \mathbf{z}_{e}^{(m, s)}\right) \\
= & c_{l}^{(m, s)} \alpha_{f, \eta}(p) a(p)+i_{f, \eta}^{(m, s)}(p)+\hat{v}_{\eta}^{(m, s)}(p) \\
& +\sum_{e} c_{e}^{(m, s)} \hat{z}_{e, \eta}^{(m, s)}(p)
\end{aligned}
$$

where $\alpha_{f, \eta}(p)$ is given by (2.6) and

$$
\begin{aligned}
i_{f, \eta}^{(m, s)}(p)= & \frac{1}{N} \sum_{l=0}^{L-1} \sum_{\substack{k \in \mathcal{S}_{l} \\
k \neq p}} \sum_{u=0}^{U-1} \sum_{n=0}^{N-1} c_{l}^{(s)} h_{n-u, l}^{(m)}(n) \\
& \cdot e^{j\left((2 \pi / N) n\left(k-p+\eta_{l}\right)+\beta\right)} e^{-j(2 \pi / N) u k} a(k) .
\end{aligned}
$$

A single DOA estimation and beamforming processor is shared between all subarrays. DOA's of the received signal's dominant path (and possibly other secondary multipath components) are assigned to subarrays to enable computation and update of their respective weight vectors. In addition, the same weight vector may be used for more than one subarray if secondary paths are unused (or for economy of implementation). Let $\mathbf{w}_{b}^{(m)}(p) \in \mathcal{C}^{S}$ denote the $p$ th subchannel's beamforming vector for the $m$ th subarray. The $m$ th subarray output is given by

$$
\tilde{\boldsymbol{a}}^{(m)}(p)=\left[\mathbf{w}_{b}^{(m)}(p)\right]^{H} \mathbf{y}^{(m)}(p)
$$

where $\mathbf{y}^{(m)}(p)=\left[y^{(m, 0)}(p), y^{(m, 1)}(p), \cdots, y^{(m, S-1)}(p)\right]^{T}$. We formulate the solution for $\mathrm{w}_{b}^{(m)}(p)$ using the well-known generalized sidelobe canceler (GSC) framework [10]. The GSC formulation of the beamformer is particularly useful since it readily lends itself to recursive implementations using standard LMS or RLS type algorithms or via block sample covariance matrix inversion. The GSC uses a constrained output energy minimization criterion. Under a signal preserving constraint, it yields the corresponding MMSE solution for beamformer weights. The constrained optimization problem may be formulated as [10]

$$
\begin{aligned}
& \mathbf{w}_{b}^{(m)}(p)=\arg \min _{\mathbf{w}} \mathbf{w}^{H} \mathbf{R}_{y}^{(m)}(p) \mathbf{w} \\
& \text { subject to }\left[\mathbf{C}_{p}^{(m)}\right]^{H} \mathbf{w}=\mathbf{f}
\end{aligned}
$$

where $\mathbf{C}_{p}^{(m)}$ is the constraint matrix whose columns represent multiple constraints; $\mathrm{f}$ is the desired constraint response; $\mathbf{R}_{y}^{(m)}(p)=\mathbf{R}_{s}^{(m)}(p)+\mathbf{R}_{i}^{(m)}(p)+\mathbf{R}_{v}+\mathbf{R}_{z}^{(m)}(p) ; \mathbf{R}_{y}^{(m)}(p) \epsilon$ $\mathcal{C}^{S \times S}$; is the $p$ th bin's received data covariance matrix for the $m$ th subarray; and $\mathbf{R}_{s}^{(m)}(p), \mathbf{R}_{i}^{(m)}(p), \mathbf{R}_{v}=\sigma_{v}^{2} \mathbf{I}_{S}$, and $\mathbf{R}_{z}^{(m)}(p)$ denote the corresponding signal, ICI, additive noise, and CCI covariance matrices, respectively. The GSC solution to (3.4) is well known [10], [26] and is given by $\mathbf{w}_{b}^{(m)}(p)=\mathbf{w}_{q}^{(m)}(p)-\mathbf{C}_{p, a}^{(m)} \mathbf{w}_{a}^{(m)}(p)$, where

$$
\begin{aligned}
\mathbf{w}_{a}^{(m)}(p)= & \left(\mathbf{C}_{p, a}^{(m)} \mathbf{R}_{y}^{(m)}(p) \mathbf{C}_{p, a}^{(m)}\right)^{-1} \mathbf{C}_{p, a}^{(m)^{H}} \\
& \cdot \mathbf{R}_{y}^{(m)}(p) \mathbf{w}_{q}^{(m)}(p)
\end{aligned}
$$

$\mathbf{w}_{q}^{(m)}(p)=\mathbf{C}_{p}^{(m)}\left[\mathbf{C}_{p}^{(m)^{H}} \mathbf{C}_{p}^{(m)]^{-1}} \mathbf{f}\right.$, and $\mathbf{C}_{p, a}^{(m)} \in \mathcal{C}^{S \times S_{C}}$ is the matrix spanning the null space of $\mathbf{C}_{p}^{(m)}$, where $S_{C}<S$ is the number of constraints used. If only one signal preserving constraint is used, $\mathbf{C}_{p}^{(m)}=\mathbf{d}^{m}(p)$ and $\mathbf{f}=1$, where $\mathbf{d}^{m}(p)$ denotes the chosen estimated steering vector of the desired user. The OSINR with the $m$ th beamformer is given as

$$
\begin{aligned}
& \operatorname{OSINR}^{(m)}(p) \\
& =\frac{\left[\mathbf{w}_{b}^{(m)}\right]^{H}(p) \mathbf{R}_{s}^{(m)}(p) \mathbf{w}_{b}^{(m)}(p)}{\left[\mathbf{w}_{b}^{(m)}\right]^{H}(p)\left(\mathbf{R}_{i}^{(m)}(p)+\mathbf{R}_{v}+\mathbf{R}_{z}^{(m)}(p)\right) \mathbf{w}_{b}^{(m)}(p)}
\end{aligned}
$$

Expressions for $\mathbf{R}_{s}^{(m)}(p), \mathbf{R}_{i}^{(m)}(p)$, and $\mathbf{R}_{z}^{(m)}(p)$ appear in the Appendix. To determine the performance gain that may be obtained from diversity combining, let the average signal-tonoise ratio (SNR) per bit at each subarray output be denoted by $\bar{\gamma}_{b}$. Assuming uncorrelated Rayleigh distributed received signals, the average probability of bit error $\left(\bar{P}_{b}\right)$ for coherent PSK subsymbols using MRC is given by [16]

$$
\bar{P}_{b, M R C}=\left[P\left(\bar{\gamma}_{b}\right)\right]^{M} \sum_{m=1}^{M}\left(\begin{array}{c}
M-2+m \\
m-1
\end{array}\right)\left[1+P\left(\bar{\gamma}_{b}\right)\right]^{m-1}
$$

where $P\left(\bar{\gamma}_{b}\right)$ denotes the probability of error for a specific alphabet size. For example, if the probability of error in a AWGN channel is given by $a \operatorname{erfc}\left(\sqrt{b \gamma_{b}}\right)$, then by averaging over the probability density function of $\gamma_{b}, P\left(\bar{\gamma}_{b}\right) \approx$ $a /\left(2 b \bar{\gamma}_{b}\right)$ [16]. Channel estimation for MRC is performed on post-beamforming outputs from each subarray, which greatly minimizes the impact of CCI on the channel estimates.

\section{Simulation Results}

Consider an example of a wireless OFDM system with the following parameters:

- Total number of subchannels $N=256$;

- Useful symbol time $T_{\text {sym }}=230 \mu \mathrm{s}$; 


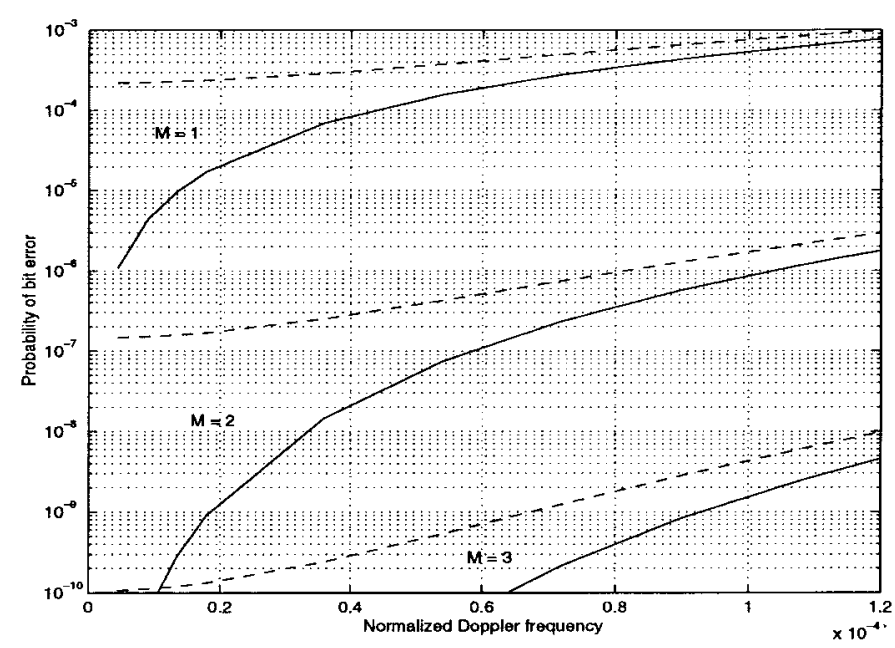

Fig. 7. $\bar{P}_{b}$ due to ICI versus normalized Doppler frequency spread for $M=$ 1,2 , and 3 using MRC. Maximum normalized frequency offset $\eta_{\max }=0$ (solid lines) and 0.02 (dashed lines). Other parameters specified in Section IV.

- $\mathrm{CP}$ or guard time $T_{C P}=20 \mu \mathrm{s}$;

- symbol rate $f_{\text {sym }}=1 /\left(T_{\text {sym }}+T_{C P}\right)=4 \mathrm{KHz}$.

Thus, interchannel spacing $f_{\text {bin }}=1 / T_{\text {sym }}=4.348 \mathrm{KHz}$, and total occupied bandwidth $=N f_{\text {bin }}=1.11 \mathrm{MHz}$ (Fig. 3). Using QPSK modulated subsymbols, the aggregate data rate $=$ (2 bits/subchannel)( $N$ subchannels/symbol) $\left(f_{\text {sym }}\right.$ symbols/s) $=2.048 \mathrm{Mb} / \mathrm{s}$. For an uplink multiple access system with these parameters, let there be $L=32$ independent users, each with $K=8$ subchannels, resulting in the allocation of a raw bit rate of $64 \mathrm{~Kb} / \mathrm{s}$ per user. Other factors that can reduce the user available data rate or the number of usable bins include analog and digital filtering constraints, spectral mask requirements, and bandwidth overhead for control and signaling.

Consider first the effect of ICI only without additive noise and CCI. In Fig. 7, the average probability of bit error $\left(\bar{P}_{b}\right)$ is plotted versus $f_{m} / f_{s}$ for $\eta_{\max }=0$ and 0.02 , respectively. It is seen that $M$-branch spatial diversity using MRC ( $M=$ 2,3 ) is very effective. This result is not surprising since ICI is spatially and spectrally distributed and MRC is known to be the optimum array combining method in the presence of noise only.

Consider now the performance of maximum SINR optimum array combining for CCI suppression on a slowly time-varying channel. Using (3.5) and (3.6), Fig. 8 plots OSINR versus relative CCI power for different number of array elements and three co-channel interferers. SNR due to AWGN and ICI is $15 \mathrm{~dB}$. Each point is obtained after averaging over 200 independent trials. As expected, maximum SINR combining (solid lines) outperforms MRC combining (dashed lines) whenever $M$ is greater than the number of interferers. For $M=$ 2, both MRC and maximum SINR combining yield similar performance, and a 3-dB array processing gain is obtained for AWGN only (when CCI is negligible). Similar results are obtained in Fig. 9, where the number of array elements is fixed at $M=6$, while the number of equal powered CCI interferers is varied as $Z=2,4$, and 8 .

For fast time-varying fading channels, Figs. 10 and 11 depict the performance of the two-stage beamforming and

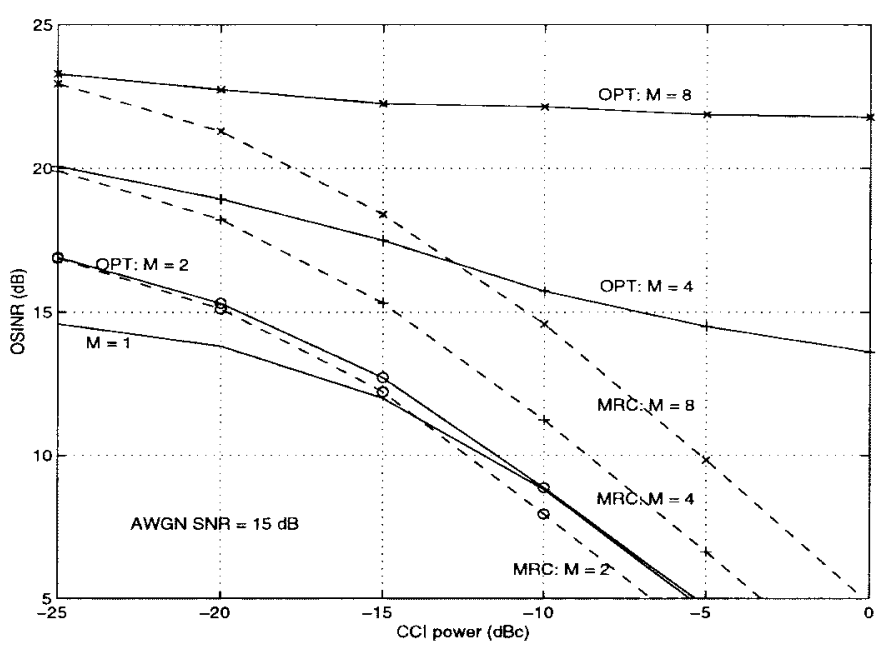

Fig. 8. CCI suppression with maximum SINR array combining (solid lines) and MRC combining (dashed lines) on a slow time-varying fading channel. OSINR is plotted versus relative CCI power with background SNR due to $\mathrm{AWGN}=15 \mathrm{~dB}$. CCI arises from three equal power co-channel interferers for array with $M=2,4$, and 8 elements. Other parameters are specified in Section IV.

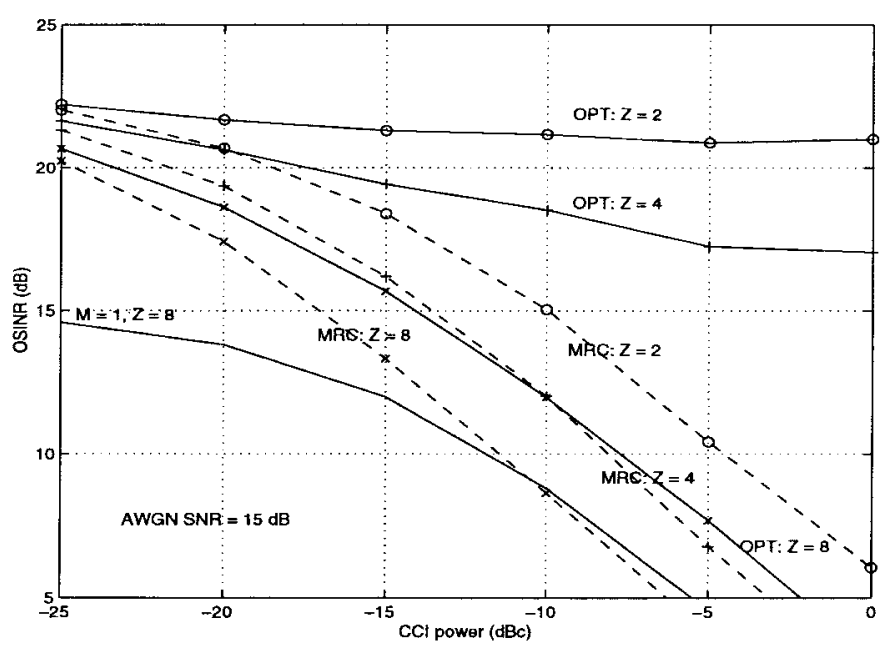

Fig. 9. CCI suppression with maximum SINR array combining (solid lines) and MRC combining (dashed lines) on a slow time-varying fading channel. OSINR is plotted versus relative CCI power with background SNR due to AWGN $=15 \mathrm{~dB}$. Number of array elements $M=6$ for $Z=2,4$, and 8 equal power co-channel interferers. Other parameters are specified in Section IV.

diversity combining method of Section III. In Fig. 10, OSINR is plotted versus relative CCI power for two-path interference for a single subarray $(M=1)$ and different number of subarray elements $(S=3,4,6,8)$. SNR due to AWGN only is taken to be $15 \mathrm{~dB}$. In Fig. 11, the probability of bit error (3.7) is plotted for coherently demodulated QPSK versus the input SINR (referred to hereafter as ISINR) with a background SNR due to AWGN and ICI of $20 \mathrm{~dB}$. Solid lines are for a single subarray $(M=1)$ with $S=3,4,6$, 8. Dashed lines are for combined beamforming and MRC with $(S=3, M=2)$ and $(S=4, M=2)$. For a given number of total elements, combined use of angle of spatial diversity is clearly superior to angle diversity alone. It is seen that diversity combining is most beneficial at relatively higher 


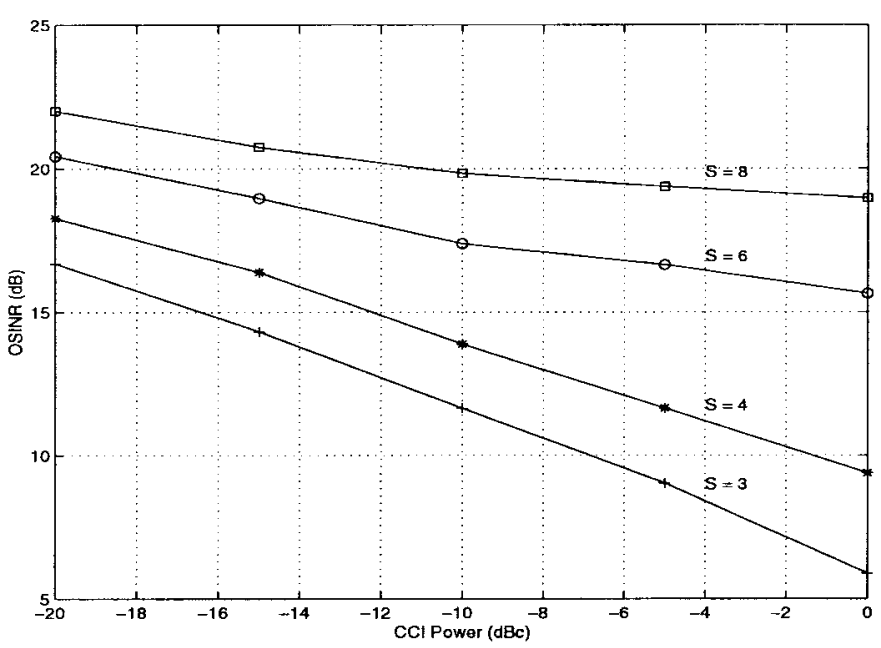

Fig. 10. OSINR versus relative CCI power for fast time-varying channel using two-stage beamforming and diversity combining with background SNR due to $\mathrm{AWGN}=15 \mathrm{~dB}$. Other parameters are specified in Section IV.

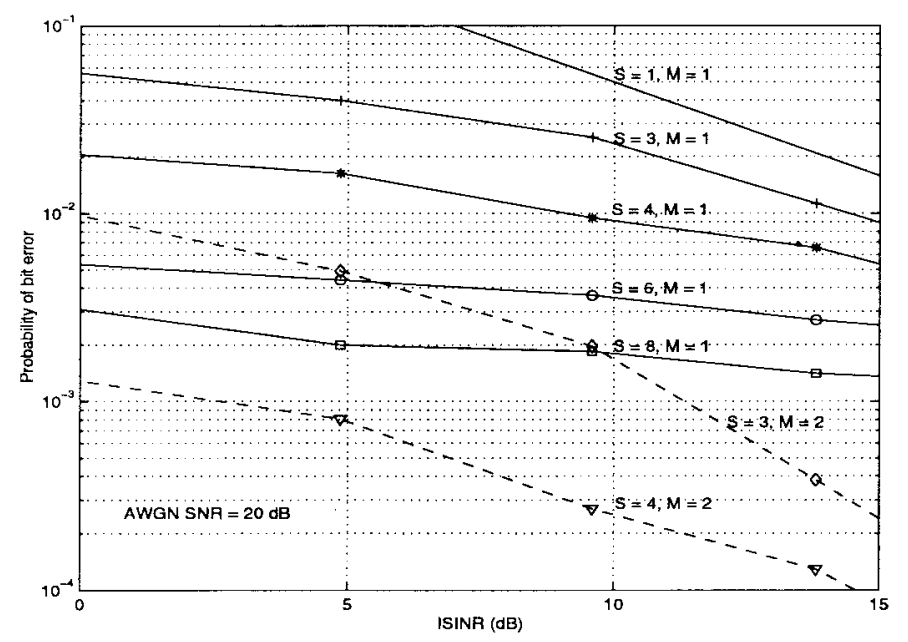

Fig. 11. $\bar{P}_{b}$ versus ISINR for fast time-varying fading channel with two-stage beamforming and diversity combining with background SNR due to $\mathrm{AWGN}=20 \mathrm{~dB}$. Other parameters arespecified in Section IV.

ISINR's [see (3.7)], whereas beamforming is most effective for relatively lower ISINR's when the interference is strong. In both figures, interelement spacing within each subarray is taken to be $\rho=\lambda / 4$, and individual subarrays are assumed to be sufficiently far apart to experience independent fading. When DOA-based beamforming is used, the dominant DOA of each user is distributed in the interval $\left[-60^{\circ},+60^{\circ}\right]$ with an angle spreading on each path of $5^{\circ}$.

The main computational load of baseband signal processing in a single-antenna OFDM receiver arises from the $N$-point FFT and the FEQ. Other algorithms requiring computation include frequency and time synchronization, channel estimation, and decoding. In the array receiver of Fig. 4, $M$ FFT's per OFDM symbol and a FEQ with $M$ taps per bin are required. Note that the $M$ FFT's may be carried out by fewer than $M$ FFT blocks using a combination of sequential and parallel execution for each OFDM symbol. Similarly, the receiver architecture in Fig. 5 requires $M S$ FFT's per OFDM symbol followed by a maximum of $(M+S)$ beamformer/diversity combiner taps per bin. Other main sources of additional complexity in the array receiver include the need for separate RF-to-baseband conversion for each antenna element, DOA processor, and channel estimation.

The use of coding has not been explicitly studied in this work. It is known that coding provides a SNR gain similar to diversity in Rayleigh fading channels [16] (proportional to minimum distance of the code) at the expense of encoding/decoding complexity and latency. A similar gain in array processing comes at the expense of increased computation and hardware due to multiple antenna elements. Thus, realistically obtainable performance gains from coding in the presence of fading only are expected to be similar to the case of diversity combining only. On the other hand, arrays are particularly well suited to improve OSINR in the presence of CCI by exploiting its spatial structure, especially via beamforming. The role of coding in this situation is unclear and needs to be further studied. In any case, the described array processing techniques do not preclude the use of popular coding techniques (convolutional, block, and combinations), and their joint use may be most attractive in systems constrained by both fading and interference. While the techniques and analysis presented in this paper are in the context of multiuser systems, they can be used in point-to-point broadband OFDM links as well. This approach is also compatible with hybrid multiple access schemes such as OFDM-TDMA and multicarrier-CDMA [11]. A natural extension of this work lies in exploring the use of adaptive arrays for OFDM systems that utilize transmitter pulse shaping, alternate transforms (for example, the discrete wavelet transform), spread spectrum modulation, or more sophisticated equalizer structures. This would lead to joint space-time-frequency OFDM receiver structures and enable superior utilization of wireless resources in these dimensions.

\section{CONCLUSIONS}

This paper has studied spatial signal processing approaches for interference suppression in wireless OFDM multiple access communication systems such as for cellular mobile radio. Interference sources considered include ICI due to fast timevarying fading and frequency offsets and CCI. For fast-fading and frequency offset induced ICI, spatial diversity using MRC is most effective. More sophisticated array combining schemes are used in the presence of CCI. For slowly time-varying fading channels, pilot assisted array combining provides superior performance compared with MRC when the number of dominant interferers is smaller than the number of array elements. Otherwise, there is no advantage of using optimum combining over MRC. Drawbacks of this approach include bandwidth overhead for pilot subsymbols and necessity for channel estimation. For fast time-varying channels, maximum SINR combining is impractical due to the low ratio of symbol rate to fading rate in OFDM systems, and a two-stage technique using constraint-based beamforming followed by diversity combining is described. As such, this method can also be used for slowly fading channels. In addition, by using differential signaling and switched diversity combining, this 
method can still provide the benefits of angle diversity while eliminating the need for channel estimation. Drawbacks of DOA-based beamforming include the computational load of DOA estimation algorithms and the need for array calibration and operation in environments where received signals are not spatially localized. Simulations show that significant gains can be realized in OSINR or probability of error improvement via array processing in wireless OFDM systems, albeit at the expense of multiple RF demodulators and additional baseband processing.

\section{APPENDIX}

Assuming uncorrelated subsymbols, $E\left[a(k) a^{*}(l)\right]=$ $E_{s} \delta(k-l)$. Normalized frequency offsets are modeled as independent and uniformly distributed each symbol time in the interval $\left[-\eta_{\max },+\eta_{\max }\right]$. Using the WSSUS property and a Rayleigh fading Doppler spectrum of the form

$$
D(f)=\frac{1}{2 \pi f_{m}}\left[1-\left(f / f_{m}\right)^{2}\right]^{-1 / 2} \quad|f|<f_{m}
$$

we have

$$
\begin{aligned}
\left.E\left[\left|\alpha_{f, \eta}(p) a(p)\right|^{2}\right]\right] & \frac{E_{s}}{N^{2}} \sum_{n=0}^{N-1} \sum_{n^{\prime}=0}^{N-1} E\left[\sum_{u=0}^{U-1} h_{n-u, l}(n) h_{n^{\prime}-u, l}^{*}\left(n^{\prime}\right)\right] \\
& \cdot E\left[e^{j(2 \pi / N)\left(n-n^{\prime}\right) \eta_{l}}\right] \\
= & \frac{E_{s}}{N^{2}} \sum_{n=0}^{N-1} \sum_{n^{\prime}=0}^{N-1} J_{0}\left(2 \pi\left(n-n^{\prime}\right) f_{m} / f_{s}\right) \\
& \cdot\left[\frac{\sin \left(\eta_{\max } 2 \pi\left(n-n^{\prime}\right) / N\right)}{\left(\eta_{\max } 2 \pi\left(n-n^{\prime}\right) / N\right)}\right] .
\end{aligned}
$$

Similarly, with uncorrelated CCI

$$
\begin{aligned}
& E\left[\left|i_{f, \eta}(p)+\hat{z}_{\eta}(p)\right|^{2}\right] \\
& =\frac{E_{s}}{N^{2}} \sum_{l=0}^{L-1} \sum_{\substack{k \in \mathcal{S}_{l} \\
k \neq p}} \sum_{n=0}^{N-1} \sum_{n^{\prime}=0}^{N-1} \\
& \cdot E\left[\sum_{u=0}^{U-1} h_{n-u, l}(n) h_{n^{\prime}-u, l}^{*}\left(n^{\prime}\right)\right] \\
& \text { - } E\left[e^{j(2 \pi / N)\left(n-n^{\prime}\right) \eta_{l}}\right] e^{-j(2 \pi / N)\left(n-n^{\prime}\right)(k-p)} \\
& +E\left[\left|\hat{z}_{\eta}(p)\right|^{2}\right] \\
& =\frac{E_{s}}{N^{2}} \sum_{\substack{k=0 \\
k \neq p}}^{N-1} \sum_{n=0}^{N-1} \sum_{n^{\prime}=0}^{N-1} J_{0}\left(2 \pi\left(n-n^{\prime}\right) f_{m} / f_{s}\right) \\
& \cdot\left[\frac{\sin \left(\eta_{\max } 2 \pi\left(n-n^{\prime}\right) / N\right)}{\left(\eta_{\max } 2 \pi\left(n-n^{\prime}\right) / N\right)}\right] e^{-j(2 \pi / N)\left(n-n^{\prime}\right)(k-p)} \\
& +\sum_{n=-(N-1)}^{N-1}(1-|n| / N) r_{z}(n) e^{-j(2 \pi / N) n\left(p+\eta_{l}\right)}
\end{aligned}
$$

If $\mathbf{d}^{m}(p) \in \mathcal{C}^{S}$ is the steering vector for the transmitted signals for the $p$ th bin chosen for the $m$ th subarray, then

$$
\begin{aligned}
\mathbf{R}_{s}^{(m)}(p)= & \mathbf{d}^{m}(p)\left[\mathbf{d}^{m}(p)\right]^{H} E\left[\left|\alpha_{f, \eta}(p) a(p)\right|^{2}\right] \\
\mathbf{R}_{i}^{(m)}(p)= & \frac{E_{s}}{N^{2}} \sum_{l=0}^{L-1} \sum_{\substack{k \in \mathcal{S}_{l} \\
k \neq p}} \mathbf{d}^{m}(k)\left[\mathbf{d}^{m}(k)\right]^{H} \\
& \cdot \sum_{n=0}^{N-1} \sum_{n^{\prime}=0}^{N-1}\left\{J_{0}\left(2 \pi\left(n-n^{\prime}\right) f_{m} / f_{s}\right)\right. \\
& \cdot\left[\frac{\sin \left(\eta_{\max } 2 \pi\left(n-n^{\prime}\right) / N\right)}{\left(\eta_{\max } 2 \pi\left(n-n^{\prime}\right) / N\right)}\right] \\
& \left.\cdot e^{-j(2 \pi / N)\left(n-n^{\prime}\right)(k-p)}\right\} .
\end{aligned}
$$

Similarly, if the steering vector for the eth CCI component is given by $\mathbf{d}_{e}^{m}$

$$
\mathbf{R}_{z}^{(m)}(p)=\sum_{e} \mathbf{d}_{e}^{m}\left[\mathbf{d}_{e}^{m}\right]^{H} E\left[\left|\hat{z}_{\eta}(p)\right|^{2}\right]
$$

\section{REFERENCES}

[1] S. Anderson, M. Millnert, M. Viberg, and B. Wahlberg, "An adaptive array for mobile communication systems," IEEE Trans. Veh. Technol., vol. 40, pp. 230-236, Feb. 1991.

[2] P. W. Baier, P. Jung, and A. Klein, "Taking the challenge of multiple access for third generation cellular mobile radio systems-A European view," IEEE Commun. Mag., vol. 34, Feb. 1996.

[3] J. J. Beek, M. Sandell, and P.O. Borjesson, "ML estimation of time and frequency offset in OFDM systems," IEEE Trans. Signal Processing, vol. 45, pp. 1800-1805, July 1997.

[4] J. A. Bingham, "Multicarrier modulation: An idea whose time has come," IEEE Commun. Mag., pp. 5-14, May 1990.

[5] L. J. Cimini, "Analysis and simulation of a digital mobile channel using orthogonal frequency division multiplexing," IEEE Trans. Commun., vol. COMM-33, pp. 665-675, July 1985.

[6] A. K. Djedid and M. Fujita, "Adaptive array sensor processing applications for mobile telephone communications," IEEE Trans. Veh. Technol., vol. 45, pp. 405-416, Aug. 1996.

[7] B. Engstrom and C. Ostberg, "A system for test of multi-access methods based on OFDM," in Proc. Vehic. Technol. Conf., Stockholm, Sweden, June 1994, pp. 1843-1845.

[8] V. K. Garg and L. Huntington, "Application of adaptive array antenna to a TDMA cellular/PCS system," IEEE Commun. Mag., pp. 148-152, Oct. 1997.

[9] L. C. Godara, "Applications of antenna arrays to mobile communications, Part I: Performance improvement, feasibility and system considerations," Proc. IEEE, vol. 85, pp. 1031-1060, July 1997.

[10] S. Haykin, Adaptive Filter Theory. Englewood Cliffs, NJ: PrenticeHall, 1996.

[11] P. Jung et al., "On Multicarrier CDMA mobile radio systems with joint detection and coherent receiver antenna diversity," Int. J. Wireless Inform. Networks, vol. 4, no. 2, Apr. 1997.

[12] S. Kapoor, D. J. Marchok, and Y. F. Huang, "Pilot assisted synchronization in wireless OFDM systems over fast fading channels," Proc. Vehic. Technol. Conf., Ottawa, Ont., Canada, May 1998.

[13] J. Kennedy and M. C. Sullivan, "Direction finding and "Smart antennas" using software radio architectures," IEEE Commun. Mag., pp. 62-68, May 1995.

[14] W. C. Jakes, Microwave Mobile Communication. New York: IEEE, 1994.

[15] R. O'Neill and L. B. Lopes, "A study of novel OFDM transmission schemes for use in indoor environments," in Proc. Vehic. Techniol. Conf., Atlanta, GA, June 1996, pp. 909-913.

[16] K. Pahalvan and A. Levesque, Wireless Information Networks. New York: Wiley, 1995 
[17] T. S. Rappaport, J. H. Reed, and B. D. Woerner, "Position location using wireless communications on highways of the future," IEEE Commun. Mag., pp. 33-41, Oct. 1996.

[18] C. Reiners and H. Rohling, "Multicarrier transmission technique in cellular mobile communication systems," in Proc. Vehic. Technol. Conf., Stockholm, Sweden, June 1994, pp. 1645-1649.

[19] J. Rinne and M. Renfors, "An improved equalizing scheme for OFDM systems for time-variant channels," in Proc. Globecom, Singapore, Nov. 1995, pp. 879-883.

[20] H. Rohling and R. Grunheid "Performance of an OFDM-TDMA mobile communication system," in Proc. Vehic. Technol. Conf., Atlanta, GA, June 1996, pp. 1589-1593.

[21] M. Russell and G. L. Stuber, "Terrestrial digital video broadcasting for mobile reception using OFDM," Wireless Personal Commun., vol. 2, pp. 45-66, 1995.

[22] J. Salz and J. H. Winters, "Effect of fading correlation on adaptive arrays in digital mobile radio," IEEE Trans. Veh. Technol., vol. 43, pp. 1049-1057, Nov. 1994

[23] G. Santella, "Bit error rate performances of M-QAM orthogonal multicarrier modulation in the presence of time-selective multipath fading," in Proc. ICC, Seattle, WA, 1995.

[24] S. C. Swales, M. A. Beach, D. J. Edwards, and J. P. McGeehan, "The performance enhancement of multibeam adaptive base-station antennas for cellular land mobile radio systems," Proc. IEEE Trans. Veh. Technol., vol. 39, pp. 56-67, Feb. 1990

[25] L. Tomba and W. A. Krzymien, "Effect of carrier phase noise and frequency offset on the performance of multicarrier CDMA systems," in Proc. ICC, Dallas, TX, 1996, pp. 1513-1517.

[26] B. D. Van Veen and K. M. Buckley, "Beamforming: A versatile approach to spatial filtering," IEEE Acoust., Speech, Signal Processing Mag., vol. 5, pp. 4-24, Apr. 1988.

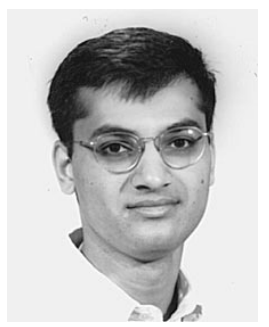

Samir Kapoor (M'98) received the B.Tech. degree from the Indian Institute of Technology, Bombay, in 1992, the M.S. degree from Washington State University, Pullman, in 1994, and the Ph.D. degree from the University of Notre Dame, Notre Dame, IN, in 1998, all in electrical engineering.

From 1994 to 1998, he was with Tellabs Research Center, Mishawaka, IN. He is currently with NEC USA, Inc., Princeton, NJ. His research interests include digital communications, adaptive signal processing, and array processing.

Dr. Kapoor is a member of Tau Beta Pi and Eta Kappa Nu and currently serves an Associate Editor for the IEEE Transactions on Multimedia.

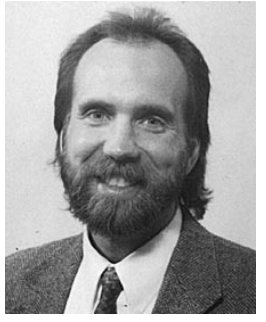

Daniel J. Marchok received the B.S and M.S. degrees in electrical engineering from Purdue University, West Lafayette, IN, in 1985 and 1987, respectively.

He currently works at the Tellabs Research Center for Tellabs Operations, Inc., Mishawaka, IN, as a Senior Research Engineer, where he leads a group of researchers in the signal processing area. His current research areas include broadband modem design for cable, xDSL, and wireless modem applications, echo cancellation in nonlinear environments, noise cancellation, and timing and synchronization algorithms. Prior research areas have included multicast switch architectures and ATM. He also holds the position of Adjunct Professor at Andrews University, Berrien Springs, MI, where he has been involved in various capacities since 1988 . He has also served as the School Board Chairman for South Bend Junior Academy, South Bend, IN, since 1998 .

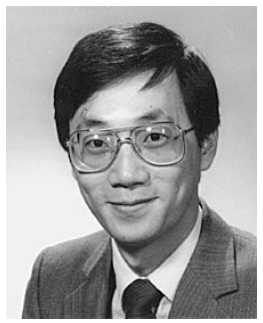

Yih-Fang Huang (F'95) received the B.S. degree from National Taiwan University, Taipei, Taiwan, R.O.C., in 1976, the M.S. degree from the University of Notre Dame, Notre Dame, IN, in 1980, and the Ph.D. degree from Princeton University, Princeton, NJ, in 1982, all in electrical engineering.

Since August 1982, he has been on the faculty at the University of Notre Dame, where he is currently Professor and Chair of the Electrical Engineering Department. In Spring 1993, he received the Toshiba Fellowship and was Toshiba Visiting Professor at Waseda University, Tokyo, Japan, within the Department of Electrical Engineering. His research interests are in the general area of adaptive signal processing with applications to communications. He has been one of the leading contributors to the field of set-membership parameter estimation and has made fundamental contributions to this field with his OBE algorithms, which led to a novel adaptive equalization paradigm, namely, the updatorsharing adaptive parallel equalizer (U-SHAPE). With the collaborations of his students, he also developed set-membership adaptive recursive techniques (SMART), which is a toolbox for set-membership filtering. Currently, his primary interests are in applications of SMART and other adaptive techniques to interference mitigation in wireless communications.

Dr. Huang served as Associate Editor for the IEEE TRANSACTIONS ON Circuits AND Systems from 1989 to 1991 and again from 1992 to 1993. Currently, he is an Associate Editor for the Journal of the Franklin Institute and a member of the Steering Committee of the IEEE TRANSACTIONS ON Multimedia. He served as Vice President-Publications for the IEEE Circuits and Systems Society from January 1997 to December 1998. 